\title{
An Endogenous Gircadian Rhythm in Neurospora crassa
}

\author{
By D. E. BIANCHI \\ Department of Biology, San Fernando Valley State College, Northridge, \\ California, U.S.A.
}

(Received 23 December 1963)

SUMMARY

A new isolate of Neurospora crassa displays a rhythm in its growth pattern. As the mycelium grows along the surface of the medium it begins, after the 12th hr, to form aerial hyphae at the growing front. This aerial growth continues until the 24th hr of growth and then stops. Surface growth continues until about the 32nd $\mathrm{hr}$, when aerial hyphae are again formed. This alternation of surface and aerial hyphae results in a colony with zones or bands of mycelia of different densities. The rhythm is circadian, about $24 \mathrm{hr}$, and is perpetuated under conditions of constant light, temperature and humidity. The rhythm has remained steady in continuous darkness for over 2 months. Banding can be somewhat influenced by temperature and light but is not synchronized by these factors. A heat sensitive factor can be isolated from the growth medium which will eliminate the rhythm.

\section{INTRODUCTION}

Periodicity of growth and reproduction in fungi has been described repeatedly in the last 60 years (Brandt, 1953). Most of these reports of the formation of zones in developing colonies indicate that the zones are caused by a repetitive oscillation of an environmental factor. However, a substantial number of reports suggest that an endogenous clock may direct the rhythm. To show that a clock is endogenous, it is necessary to grow the culture under constant conditions and for it to continue to demonstrate the periodicity. The periodicity must also have a frequency which deviates from other periodicities in the environment such as earth-rotation or tidal rhythm (Aschoff, 1960). Most plant rhythms damp out when kept for long periods of time under constant conditions (Bruce, 1960). This is true for the reported cases of periodicity in Neurospora crassa (Brandt, 1953; Pittendrigh et al. 1959). The periodicity in $N$. crassa (strain Sussman) reported in this paper does not damp out under the constant environmental conditions tried. The periodicity is also shown to be endogenous and circadian (about $24 \mathrm{hr}$ ) as defined by Halberg (1959).

The rhythm in the strain of Neurospora crassa (Sussman) used in the present work is expressed both in growth, as measured by the density of the mycelial mat, and reproduction. About every $24 \mathrm{hr}$ there is an increase in the branching of the mycelium and the formation of conidiophores. The resulting appearance of the colony is a series of concentric circles when grown on a Petri plate, or of bands when grown along tubes. 


\section{METHODS}

The strain of Neurospora crassa used in these experiments was obtained from Dr A. Sussman (University of Michigan, Ann Arbor, Michigan) who originally isolated it.

The stock culture is maintained on a defined medium consisting of ammonium tartrate, $5 \cdot 0$ g.; $\mathrm{NH}_{4} \mathrm{NO}_{3}, 1 \cdot 0$ g.; $\mathrm{KH}_{2} \mathrm{PO}_{4}, 1 \cdot 0$ g.; $\mathrm{MgSO} .7 \mathrm{H}_{2} \mathrm{O}, 0 \cdot 5$ g.; $\mathrm{NaCl}, 0 \cdot 1 \mathrm{~g}$; $\mathrm{CaCl}_{2} .2 \mathrm{H}_{2} \mathrm{O}, 0.1 \mathrm{~g}$; sucrose, $10 \cdot 0$ g.; biotin, $4.0 \mu \mathrm{g}$.; trace element solution (Ryan, 1950), $1.0 \mathrm{ml}$; distilled water, 1.0 l.; and agar, $15.0 \mathrm{~g}$; adjusted to $\mathrm{pH} 5.5-5.8$ before autoclaving. The stock cultures used for inoculation were maintained at $25^{\circ}$ and transferred so that all inocula were 3 days old. This same defined medium was used for all experimental work except where stated otherwise. Liquid cultures were prepared in the same manner except that agar was omitted.

The cultures were incubated either in a light-tight, controlled-temperature incubator, or in a controlled-temperature room equipped with a single light source at one end of the room. The temperature of this room was maintained at $26^{\circ} \pm 1$. Humidity and temperature were recorded during all experiments by a recording hygrothermograph. Barometric pressure was recorded by a recording aneroid barometer.

Light for the photo-experiments was provided by 'cool white' fluorescent tubes. Circulation of air in the controlled-temperature room prevented the formation of a measurable thermal gradient between the lamp and the cultures growing at various distances from the lamp. For experiments with selected wavelengths of light, the growth tubes were covered with gelatin filters obtained from Bates Lighting and Scenic Studios, Hollywood, California. The absorbance of these filters was measured in a DU-spectrophotometer (Beckman Instruments, Inc., 2500 Harbor Blvd., Fullerton, California). The red filter (Roscoe, No. 22) absorbed $95 \%$ or greater of the radiation between 500 and $560 \mathrm{~m} \mu$ and below $340 \mathrm{~m} \mu$. The yellow filter (Roscoe No. 3) absorbed $95 \%$ or greater of the radiation between 370 and $460 \mathrm{~m} \mu$ and the blue filter (Roscoe No. 129) absorbed $95 \%$ or greater of the radiation above $540 \mathrm{~m} \mu$, and below $340 \mathrm{~m} \mu$. Measurements of light intensity were made in foot candles with a Weston foot-candle meter (Model 614).

\section{RESULTS}

\section{Description of the growth rhythm}

After inoculation of the culture on a complete medium, growth was first characterized by the appearance of filaments growing across the surface of the medium from the point of inoculation. These filaments branched repeatedly as they grew, but tended to stay on the surface of the medium for the first $12 \mathrm{hr}(A$ to $B, \mathrm{Pl}$. 1, fig. 1). Because of repeated branching of the mycelium as it progressed outward from the point of inoculation, a colony became more and more dense towards its periphery. Starting at about the 12-14 hr of growth, $B$, hyphae began to grow upward, forming aerial hyphae at the leading edge of the culture. Thus, during the next $8 \mathrm{hr}(B$ to $C$ ) the culture formed, at its circumference, a dense mat of branched hyphae, some of which were aerial, some on the surface, and a few growing 
into the medium. In some instances the aerial hyphae formed conidiophores. At about $24 \mathrm{hr}$, growth appeared to stop $(C)$, and then from the periphery of the dense growth appeared a few hyphae growing along the surface of the medium. Growth did not actually stop, as was shown in later experiments. As these new surface hyphae grew, they again began to branch and a new band was formed.

The pattern of growth described above is what is usually seen when the cultures are grown in the laboratory, but the variations in this pattern are numerous. After inoculation there may be a delay of several days before growth or periodicity are noticed. The $\mathbf{2 4} \mathrm{hr}$ cycle may vary by several hours and the uniformity of the bands may also break. Most of these variations could be diminished by careful preparation of the growth medium, such as duration of autoclaving, adjustment of the $\mathbf{p H}$ value and addition of equivalent amounts of medium in each tube, as well as growth under controlled conditions.

\section{Growth under controlled conditions}

The strain of Neurospora used grows at a relatively rapid rate on the medium described. This made it impractical to use Petri plates and hence all experiments were performed with $30 \mathrm{~cm}$. long 'race track' tubes $(1 \mathrm{~cm}$. diameter glass tubing with the ends turned up at a $45^{\circ}$ angle). Inoculation was made at one end of the tube, with a sample from a suspension of hyphae in sterile water. After inoculation, the tubes were placed in the dark until growth and banding were detectable and then transferred to the experimental chambers. Measurement and timing were made only after the second band was formed.

By these procedures, cultures were set up and put under two constant environmental conditions. The first set of cultures was put in a constant temperature room at $26^{\circ} \pm 1^{\circ}$ in the dark. The second set of cultures was placed in a constant temperature room, also at $26^{\circ} \pm 1^{\circ}$, but with constant illumination. The average light intensity at the site of the cultures was 100 foot candles. The humidity was not controlled but was recorded and did not vary more than $\pm 5 \%$ from an average of $45 \%$. The cultures were maintained for two months under these conditions. The cultures grown in the dark were only exposed to light during subculture; these exposures were brief and less than $1 \mathrm{ft}$. candle.

During this 2-month period, the periodicity of density in the mycelium in both illuminated and non-illuminated cultures continued without any indication of a loss in response, with a periodicity of about $24 \mathrm{hr}$. The period might vary on any one day by as much as $3 \mathrm{hr}$. Thus, it appears that the degree of illumination and the temperature used in these experiments did not induce or synchronize the periodicity. The variations in humidity were not regular or in cycles of $24 \mathrm{hr}$. The variations in the barometric pressure also were not rhythmical. The possibility of some unknown environmental synchronizer is lessened by the fact that when several culture tubes were inoculated at the same time there was no tendency for all of the cultures to form bands at the same time of the day, although the 24-hr periodicity of such cultures did remain constant.

Since it was not easily possible to determine whether growth stopped entirely after the formation of $C$ (PI. 1, fig. 1) and before the initiation of a new band, timelapse photography was used, one frame being exposed every 85 sec. After development, the film was projected on a large sheet of white paper and advanced normally. 
Each 25 frames (35 min., 25 sec.) the advancing border of the colony was marked on the paper. The results (Fig. 1) indicate that the rate of linear surface growth was constant. The formation of the bands was thus the result of periodic development of aerial hyphae and conidiophores.

\section{Initiation of the rhythm}

When hyphae were transferred from an actively-growing colony to fresh media, a new cycle of banding began. To determine whether the developmental stage of the inoculum had any effect on the initiation of the subsequent bands, experiments were done in which hyphae were removed for inoculation from each of the regions $A, B$, and $C(P 1.1$, fig. 1). Small blocks of medium were transferred and the culture incubated in the dark at $20^{\circ}$. In each case, the time and distance between the inoculum and the end of the first band was about the same. Differences in time between the formation of subsequent bands were no greater than an hour.

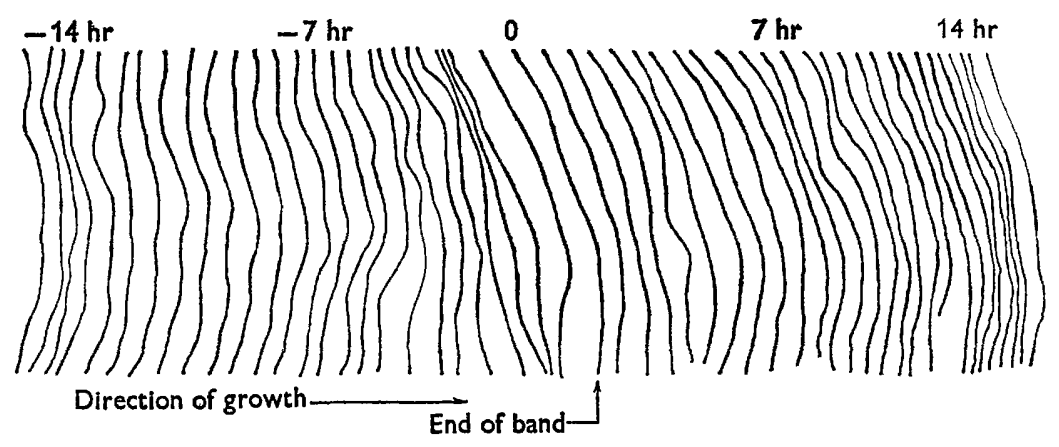

Fig. 1. Outlines of a portion of the leading edge of the colony of Neurospora crassa (Sussman) at intervals of $35 \mathrm{~min} .25 \mathrm{sec}$. showing the relatively constant (non-rhythmical) rate of linear growth. Only one band of aerial hyphae was formed during this sequence; the end of which is indicated at 0 . Times are marked relative to the termination of aerial growth.

Since factors associated with rotation of the earth, such as the hour of the day, might induce or influence banding, experiments were made in which sets of cultures were inoculated at $2 \mathrm{hr}$ intervals for $10 \mathrm{hr}$. The size of inoculum was standardized by making a suspension of hyphae in sterile water and reading the turbidity of the suspension in a colorimeter and adjusting to a known value. The cultures were incubated in the dark in the $20^{\circ}$ controlled-temperature chamber. The results are shown in Fig. 2. Banding appeared to be induced at the time of inoculation and was not synchronized, if at all influenced, by external circadian factors. A pronounced variation was also found between similar tubes inoculated at the same time.

\section{Effect of the growth medium}

The composition of the growth medium was critical in expression of the zonation in the strains of Neurospora studied by Brandt (1953). The effect on zonation of changes in the composition of the minimal medium was studied with the Sussman strain of Neurospora. The carbohydrate source in the minimal medium (sucrose) was replaced by glucose, galactose, mannose, lactose or sorbose. Growth and banding 
occurred with each of the sugars, except sorbose where no growth occurred. In a medium containing sucrose and sorbose, normal growth and banding occurred.

The minimal medium was also altered by changing the nitrogen source (both the ammonium tartrate and ammonium nitrate at the same time); in these experiments, peptone or mixtures of amino acids were used. Although the rate of growth was altered by the presence of the constituents, the pattern of banding was not affected.

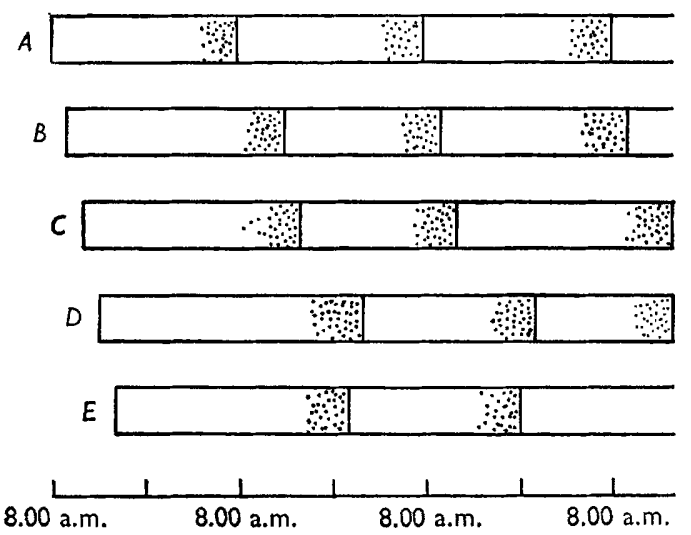

Fig. 2. Diagram showing the effect of time of inoculation on the subsequent hour of band formation. Tube $A$ was inoculated at 8.00 a.m.; each subsequent tube was inoculated at $2 \mathrm{hr}$ intervals.

Table 1. The effect of temperature on extent of linear growth and the number of bands on Neurospora crassa (Sussman). All cultures were grown on defined media for 19 days in growth tubes

\begin{tabular}{|c|c|c|c|}
\hline \multirow{2}{*}{$\begin{array}{c}\text { Tube } \\
\text { no. }\end{array}$} & \multicolumn{3}{|c|}{ Linear growth (cm.) } \\
\hline & $15^{\circ}$ & $\mathbf{2 5}^{\circ}$ & $35^{\circ}$ \\
\hline 1 & $8 \cdot 6$ & $8 \cdot 7$ & $7 \cdot 0$ \\
\hline 2 & $9 \cdot 5$ & $7 \cdot 7$ & - \\
\hline 3 & $7 \cdot 8$ & $9 \cdot 3$ & $7 \cdot 0$ \\
\hline 4 & $8 \cdot 2$ & $10 \cdot 4$ & $7 \cdot 7$ \\
\hline $\mathbf{5}$ & $10 \cdot 4$ & $10 \cdot 1$ & $6 \cdot 7$ \\
\hline 6 & $8 \cdot 0$ & $9 \cdot 2$ & $6 \cdot 9$ \\
\hline 7 & $10 \cdot 5$ & - & - \\
\hline Average & $9 \cdot 0$ & $9 \cdot 23$ & $7 \cdot 06$ \\
\hline & \multicolumn{3}{|c|}{ No. of bands } \\
\hline no. & $15^{\circ}$ & $\mathbf{2 5}^{\circ}$ & $35^{\circ}$ \\
\hline 1 & 5 & 15 & 26 \\
\hline 2 & 6 & 14 & 一 \\
\hline 3 & 5 & 15 & 23 \\
\hline 4 & 5 & 13 & 22 \\
\hline $\mathbf{5}$ & $\mathbf{5}$ & 16 & 19 \\
\hline 6 & 5 & 15 & 19 \\
\hline 7 & 6 & - & - \\
\hline Average & $5 \cdot 29$ & $14 \cdot 67$ & $21 \cdot 8$ \\
\hline
\end{tabular}




\section{Effect of temperature}

To test the effect of temperature, growth-tubes with defined medium agar were inoculated and placed in incubators at 15, 25, or $35^{\circ}$. After 19 days, the tubes were removed, and linear growth and number of bands in each tube were measured; the results are shown in Table 1 . Linear growth was not greatly different in the range $15^{\circ}$ to $35^{\circ}$, but the number of bands was appreciably altered by temperature. The $Q_{\mathbf{1 0}}$ figures for these experiments are shown in Table 2.

Table 2. The $Q_{10}$ values of linear growth and frequency of band formation in Neurospora crassa (Sussman)

\begin{tabular}{|c|c|c|c|}
\hline & & $Q_{10}$ & $\begin{array}{l}\text { Temperature } \\
\text { range }\end{array}$ \\
\hline & Linear growth* & $\begin{array}{l}1 \cdot 03 \\
0 \cdot 76\end{array}$ & $\begin{array}{l}15-25^{\circ} \\
25-35^{\circ}\end{array}$ \\
\hline & Banding $\dagger$ & $\begin{array}{l}2 \cdot 77 \\
1 \cdot 49\end{array}$ & $\begin{array}{l}15-25^{\circ} \\
25-35^{\circ}\end{array}$ \\
\hline & \multicolumn{3}{|c|}{$=\frac{\mathrm{cm} . \text { of total growth at temperature } 10^{\circ} \text { lower }}{\text {. }}$} \\
\hline$Q_{10}=$ & $\frac{\text { number of } 1}{\text { number of ban }}$ & duced & $\frac{\text { temperature }}{\text { rature } 10^{\circ} \text { low }}$ \\
\hline
\end{tabular}

\section{Effect of light}

Experiments already described indicated that light did not synchronize or initiate the banding, but did not show the influence of light on the periodicity. Experiments were made to test the effect of light intensity. At one end of a long bench in the constant temperature room, a lamp was placed with 2 15-watt 'cool white', fluorescent bulbs (General Electric F 15T8 CW). Light intensity at various distances from the light source were measured and cultures in growth tubes placed at these points. The results of these experiments are shown in Pl. 1, fig. 2. Cultures at $50 \mathrm{ft}$. c. or less grew at about the same rate or slightly faster than the cultures grown at the same temperature but in the dark. The greater the light intensity above $50 \mathrm{ft} . c$. , the less the amount of linear growth.

The cultures shown in Pl. 1, fig. 2 were photographed on the seventeenth day after inoculation and exposure to the experimental lights. Each of the tubes showed the same number of bands (16) as the tube grown in the dark. However, the tubes grown at 200, 100, and $50 \mathrm{ft} . c .$, showed a tendency to form irregular bands. During these experiments, thermometers placed at various distances from the light source showed that there was less than $\mathbf{a} .5^{\circ}$ variation in temperature between the growth tubes nearest the lamp and those furthest away.

Experiments were made to test the effect of light quality by wrapping the growth tubes with gelatin filters. The cultures grown under the red, yellow or blue filters gave the same pattern of growth and banding as shown in Pl. 1, fig. 2, even though there was not an equivalent amount of light energy produced through each filter. 


\section{An endogenous rhythm}

The experiment described above suggests that since rhythmic growth is maintained under constant environmental periodicity, that this is a truly endogenous rhythm. It is thus reasonable to envisage an internal mechanism that produces a substance as the mould grows, and which when in sufficient concentration inhibits or decreases growth; a second substance must then be produced to destroy or inactivate the first compound before growth can begin again, and the formation and inactivation of these substances must occur repeatedly as growth and banding progress.

To begin to examine these possibilities, flasks (1000 ml.) containing $200 \mathrm{ml}$. liquid growth medium were inoculated and grown as static cultures. Subsequent treatment of this growth culture is shown in the following flow diagram:

\begin{tabular}{|c|c|c|}
\hline & $\begin{array}{l}200 \mathrm{ml} \text {. of liquid defined medium } \\
\text { incubated with } N \text {. crassa } 5 \text { days } \\
\text { Growth medium separated and } \\
\text { sterilized by filtration through } \\
\text { a millipore filter }\end{array}$ & \\
\hline $\begin{array}{l}\text { Mycelial mat mixed } \\
\text { in blender with } \\
100 \text { ml. of fresh } \\
\text { defined medium } \\
\text { Autoclaved }\end{array}$ & $\begin{array}{l}\text { Growth medium mixed } \\
\text { with } 2 \% \text { agar } \\
\text { Autoclaved }\end{array}$ & $\begin{array}{l}\text { Growth medium mixed } \\
\text { sterilely with } 2 \% \text { agar }\end{array}$ \\
\hline
\end{tabular}

The growth tubes made from these media were inoculated and incubated at $20^{\circ}$ for 8 days. The results are shown in Pl. 2, figs. 3-5. The cultures grown on the mycelium supplemented medium formed bands in the usual manner. The cultures grown on the used and autoclaved culture filtrate medium showed weak evidence of banding. However, banding was not observed in the cultures grown on used culture filtrate medium sterilized by passage through the Millipore filter rather than by autoclaving. Growth under the latter conditions resulted in the continuous formation of aerial hyphae.

\section{DISCUSSION}

It is difficult to determine whether an observed rhythm in an organism is the result of an endogenous mechanism or whether this rhythm is induced or synchronized by some variable in the environment. The test is to place the organism under constant conditions and see whether the rhythm persists. The strain of Neurospora crassa (Sussman) used in these studies was put under relatively 'constant' conditions of temperature, light and humidity and the rhythm persisted. Yet there is no way of determining whether some other unconsidered environmental variable was synchronizing the changes in growth. Factors, such as pressure, varied little over longer periods of time than the $\mathbf{2 4} \mathrm{hr}$ Neurospora rhythm and thus seem unlikely synchronizers.

Since the period is circadian, it would normally be expected that if an environmental synchronizer was involved in the rhythm, the synchronizer itself would have some circadian rhythm. The result of such a system would be to time or set the 
periodicity in the mould to match that of the synchronizer. These experiments have shown, however, that there is no harmony in the cycles between the moulds inoculated at different times within a $24 \mathrm{hr}$ period. Initiation and completion of a cycle seems to be more of a function of the time of inoculation than any other factor, and there is no tendency for the cycles of moulds inoculated at different times to become synchronized with each other. The evidence seems to indicate that the observed growth rhythm in these strains of $N$. crassa are initiated and maintained within the organism.

A biological clock that is 'endogenous' does not preclude that the rhythm cannot be affected by changes in the environment. Such effects have been shown in this report by the effects of temperature and light. Temperature is a recognized factor in regulating the growth rate of many micro-organisms. The different degrees of this effect on the two aspects of growth, linear growth and banding, in this strain of Neurospora are interesting. In the temperature range $15-25^{\circ}$, the linear or forward growth of the mould down the tube was only slightly increased with increasing temperature, but in the aerial growth (that associated with the rhythm) there was almost a tripling of frequency (Table 2). High light intensity also had an effect in inhibiting linear growth but appears to play a minor role in the frequency of bands of aerial hyphae. High light intensity tends to break the uniformity of a band.

An endogenous clock is indicated from the experiments with used culture filtrate. In these experiments, it was shown that the growing mould produces a substance in the liquid growth medium which can be filtered from the mould and added to newly prepared minimal media. The presence of this filtrate in sufficient concentration will cause the mould to produce aerial hyphae at all times, thus inhibiting the rhythm. The filtrate is heat inactivated.

The nature of the mechanism is not clear. From the evidence presented in the experiments with used culture filtrate media, it seems that a possible cause is the production by the surface hyphae of an aerial activating substance which initiates the formation of aerial hyphae. The aerial hyphae, and possibly the surface hyphae also produce an inhibitor which feeds back, and when formed in great enough concentration, inhibits the action of the aerial activator. As a result, only surface hyphae grow for a time across the agar medium until the hyphae are out of range of the inhibitor and the process begins again. It is also possible that the inhibitor is only produced at periodic intervals by the surface hyphae. These possibilities are being studied.

The help of Mr Gene Kalland who performed the experiments in time lapse photography and the technical assistance of Miss Leah Imamura, are gratefully acknowledged. The author also appreciates Dr A. Sussman's advice in evaluating this study and his assistance in providing the strains. 

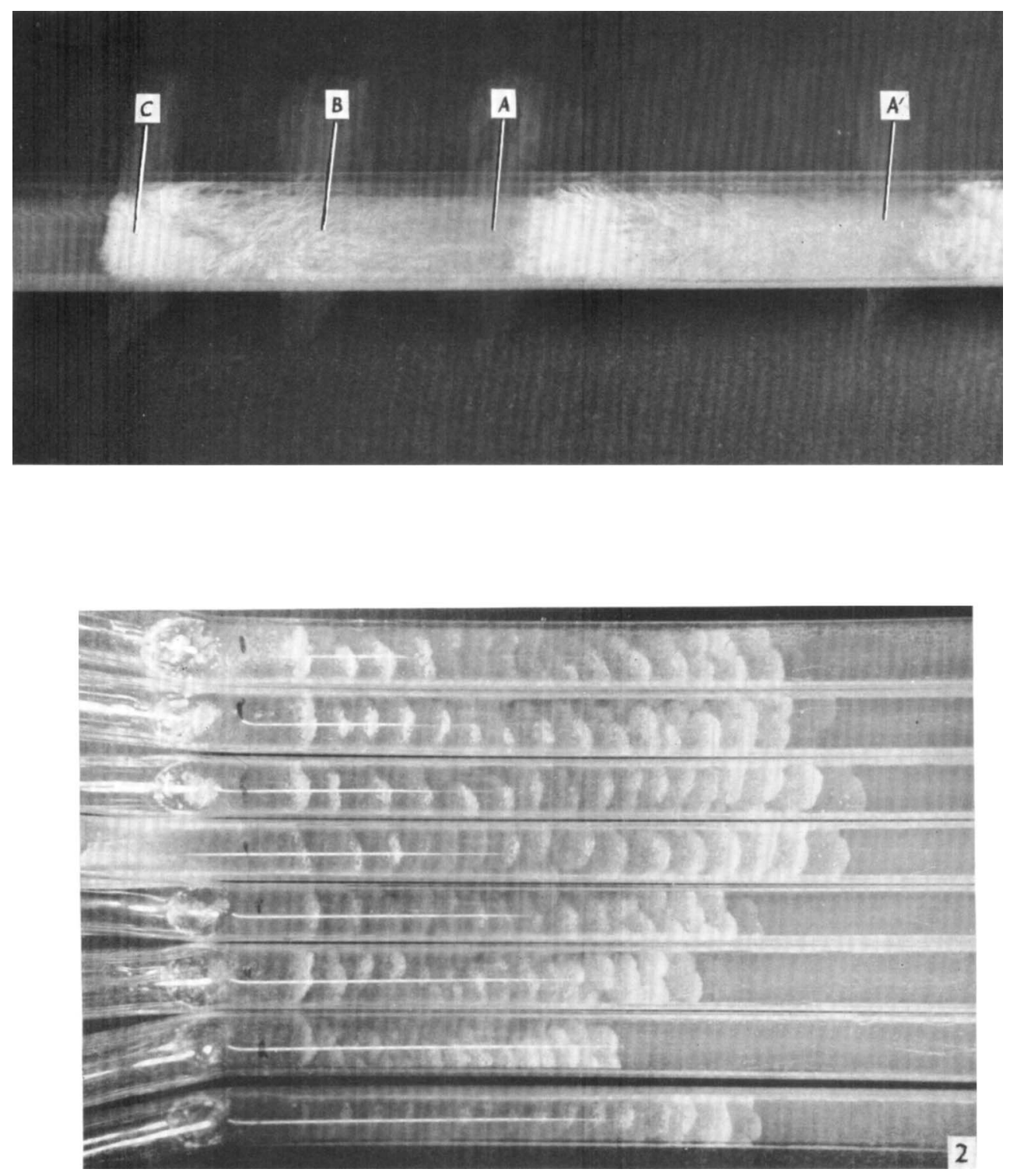

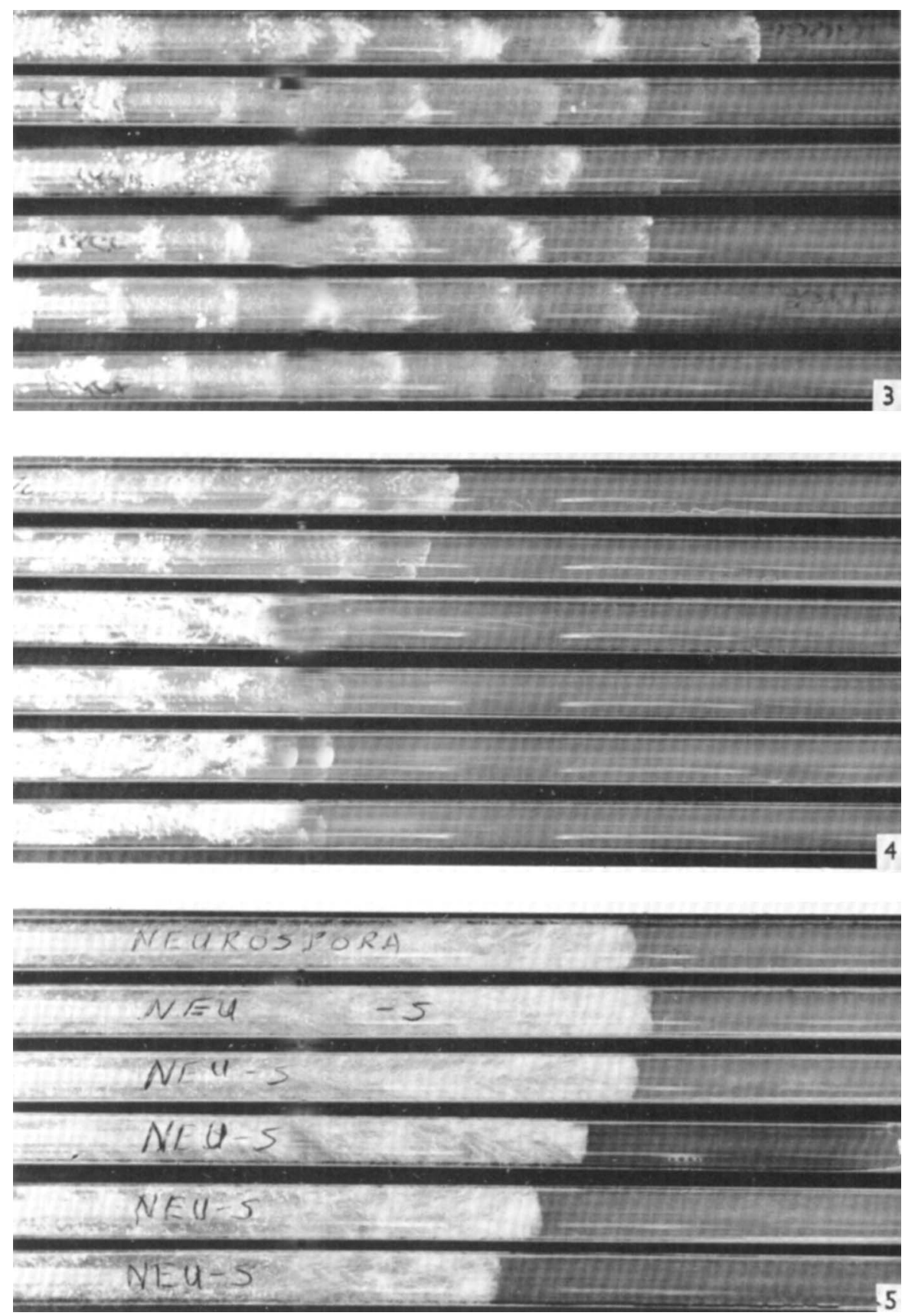

D. E. BIANCHI 


\section{REFERENCES}

Aschoff, A. (1960). Exogenous and endogenous components in circadian rhythms. Cold Spr. Harb. Symp. quant. Biol. 25, 11.

Brandt, W. H. (1953). Zonation in a prolineless strain of Neurospora. Mycologia, 45, 194. Bruce, V. G. (1960). Environmental entrainment of circadian rhythms. Cold Spr. Harb. Symp. quant. Biol. 25, 29.

Halberg, F. (1959). Physiologic 24-hr periodicity in human beings and mice, the lighting regimen and daily routine. Photoperiodism and related Phenomena in Plants and Animals, Ed. by Withrow, p. 803. Washington: American Association for the Advancement of Science.

Pittendrigh, C. S., Bruce, V. G., Rosensweig, N. S. \& Rubin, M. L. (1959). A biological clock in Neurospora. Nature, Lond. 184, 169.

Ryan, F. J. (1950). Selected methods of Neurospora genetics. Methods in Medical Research, Vol. 3. Ed. by R. W. Gerard. Chicago: 'The Year Book Publishers., Inc.

\section{EXPLANATION OF PLATES}

\section{Plate 1}

Fig. 1. Photograph of the periodic bands formed in a growth tube by the mycelium of Neurospora crassa (Sussman). One band extends from $A$ to $A^{\prime}$. $A$ and $A^{\prime}$ indicate areas of surface hyphae. $B$ indicates the area of a band where aerial hyphae are just beginning to be formed. $C$ indicates the terminal mass of aerial hyphae at the end of a band.

Fig. 2. Growth tubes showing the effect of light intensity on growth and banding. Growth at the end of the first day is marked with a crayon mark on the tube. The lower tube was grown at the same temperature but in the dark. Light intensity at each of the tubes from top to bottom is 5, $10,15,25,50,100,200$, and 0 ft.c.

\section{Plate 2}

Fig. 3. Growth of Neurospora crassa (Sussman) on medium made from fractionated mycelia and sterilized by autoclaving.

Fig. 4. Growth of Neurospora crassa (Sussman) on medium made from growth medium in which this strain of Neurospora had already grown. Medium sterilized by autoclaving.

Fig. 5. Growth of Neurospora crassa (Sussman) on medium made from growth medium in which this strain of Neurospora had already grown. Medium sterilized by filtration through a Millipore filter. 